Correspondence address: Daniel Rodger, Lecturer in Perioperative Practice, School of Health and Social Care, London South Bank University, 103 Borough Road, London, SE1 OAA. Email: daniel.rodger@Isbu.ac.uk

\title{
Extravasation injury leading to acute compartment syndrome in a child: the vital role of pulse oximetry in early detection and management
}

\section{Key Words}

Extravasation, Pulse Oximetry, Oximeter, Acute Compartment Syndrome, Paediatric, Cannulation, Perioperative

\begin{abstract}
Acute compartment syndrome as a result of an extravasation injury is rare. The perioperative environment presents a unique risk that may contribute to more serious patient outcomes. Using a case study approach we report that the placement of a pulse oximeter on the cannulated limb can function as the first sign of vascular compromise.
\end{abstract}

\section{Introduction}

This paper presents the case of a 10-year-old patient who developed acute compartment syndrome as a result of an extravasation injury during a routine urological procedure. The case describes the challenges and risk-factors associated with these types of injuries in the perioperative environment and some simple recommendations for minimising those risks. Extravasation describes intravenous (IV) fluids unintentionally escaping from the cannulation site or vein into the surrounding tissue with the potential to cause serious injury (Philips \& Gorski, 2014). Acute compartment syndrome is the rapid onset of pressure within a confined fascial compartment resulting in the blood supply to the muscle being cut off (Duckworth et al, 2017). This can lead to limb ischaemia and necrosis without urgent diagnosis and intervention to prevent permanent damage.

Extravasation leading to compartment syndrome in children is rare, however, when it does occur it presents a serious clinical complication that requires immediate diagnosis and management (Behr, 2015). Extravasation occurs in up to $11 \%$ of young children (Reynolds et al 2014) and accounts for between $23-78 \%$ of complications of peripheral intravenous (PIV) use in infants (Driscoll et al 2015). In this case report observations were made that may help to reduce the severity of acute compartment syndrome as a consequence of extravasation. Children are at significantly higher risk of a serious extravasation injury because they have multiple risk factors that make them more susceptible (Paquette et al, 2011; Treadwell 2012). When combined with a number of other salient contributing factors, the perioperative environment presents a unique risk for silent extravasation injuries. The case 
report highlights the use of a pulse oximeter on the cannulated limb was a significant factor in the early diagnosis of acute compartment syndrome resulting in a better patient outcome.

\section{Case Report}

A fit and well 10 year old boy was received into the anaesthetic room for routine urology surgery. The patient was anaesthetised using a gas induction because of high levels of anxiety. Following induction, the patient proved to be very difficult to cannulate. It took the anaesthetic consultant and registrar over one hour to site and secure a functioning cannula.

The cannula was flushed with Saline which injected easily, with no obvious signs of extravasation. The patient was then given propofol to facilitate placement of a laryngeal mask. Hartman's solution was attached to the cannula using a giving set for continuous infusion while an llio-inguinal block was performed. The patient was then brought into the operating theatre and was safely positioned, secured and draped. A wrap-around paediatric pulse oximeter was reconnected on the finger of the hand with the cannula and positioned under the drapes. The giving set was reopened and the cannula continued to be used to give IV drugs via a three-way-tap in the infusion line without undue resistance.

Fifteen minutes into the procedure the pulse oximetry trace became damped resulting in changes to the waveform and the saturations starting to decrease without any obvious ventilatory cause. The Operating Department Practitioner (ODP) went under the drapes to gain access to the hand and quickly perceived that something was seriously wrong. The hand was cold, tense and mottled. The hand was fully exposed to determine the full extent of the injury and a new cannula was sited in the foot.

The ODP then elevated the hand, squeezing out as much fluid as was possible through the cannula site while waiting for the plastic surgeon on call. The urological surgery was completed. Subsequently a plastic surgeon made multiple small incisions in the dorsum of the hand and proximal forearm, irrigating the hand with saline and hyaluronidase. The incisions were left open and bandaged. The patient's mother was informed and the patient was discharged home the following day and no further surgical intervention was necessary.

\section{Discussion}

An extravasation injury describes the damage caused by medication or fluids that have penetrated the surrounding tissue around a PIV cannula site (Al-Benna et al 2013). Extravasation is a known risk of IV drug delivery and fortunately any serious injury is uncommon. However it can result in significant complications such as skin, nerve, muscle and tendon damage, permanent paralysis and even amputation (Doellman 2009). Extravasation of a PIV cannula commonly presents with; swelling, pain, mottling, localized erythema and the cannula no longer flushing as expected or at all (Hakan \& Özer 2007). Pain caused by extravasation under anaesthesia is notoriously ambiguous as any physiological 
responses to pain might be assumed to be a response to surgical stimulus. Children are already considered to be at greater risk of extravasation injury by virtue of their smaller and more fragile veins, increased subcutaneous tissue, capillary leakage and decreased peripheral circulation (Tripi et al 2016; Paquette et al, 2011).

Extravasation in this case probably occurred shortly after transfer into the theatre and the pain associated with it was masked by the anaesthetic. Whilst the patient was positioned and waiting for surgery to begin, IV drugs and fluids were administered and flowed freely without any resistance, something which was observed in a similar case that resulted in acute compartment syndrome in the forearm following extravasation (Garg et al 2015). Although the patient in that case was an adult; there were a number of relevant similarities in regards to the presentation of the injury, lack of accessibility and visibility of the PIV cannula site. The patient responded to the urological surgical incision and additional propofol and analgesics were administered, although they had minimal effect. The patient's physiological response to pain was attributed to the surgery and an inadequate block rather than being recognised as pain caused by extravasation, and the failure of the IV drugs to reach the circulation.

Following exposure of the forearm, the IV fluids were stopped and disconnected immediately and aspiration of the residual drug was attempted using a syringe, and the cannula was then removed. The arm was then elevated to reduce congestion, a plastic surgeon was called, and manual manipulation of the limb was used to remove residual fluid through the PIV cannula site. These steps are considered to be generic steps in the immediate management of a serious extravasation injury (Goutos et al 2014).

The plastic surgical intervention included making multiple incisions around the cannula site, on the dorsal and ventral surface of the forearm and then squeezing in a distal to proximal direction followed by irrigation with saline and hyaluronidase. Extravasated drugs are diluted alleviating inflammation, chemical damage and vascular compromise.

This technique along with a number of modifications has been demonstrated to be a successful surgical treatment for serious extravasation injuries (Goutos et al 2014). When an extravasation injury has led to vascular compromise and/or the development of acute compartment syndrome then immediate surgical intervention is indicated, including the technique described (Goutos et al 2014). The earlier an extravasation injury is detected and treated - the better the outcome. Effective management of a serious extravasation injury necessitates clear communication and prompt action within the multidisciplinary team.

The nature of the intraoperative environment means that for many procedures the cannulation site is not easily accessible or visible which are known contributing factors to extravasation injuries (Major \& Huey 2016). In this case the arms were wrapped and padded and placed at the side of the patient which meant they were not easily accessible without 
interrupting the operating surgeons. Upon reflection of this case, the placement of the wrap around pulse oximeter played a significant role in the diagnosis and management of the patient's acute compartment syndrome.

Had the pulse oximeter not been on the same limb as the cannula it is possible that the extravasation injury would not have been noticed until much later when a far more serious injury would have presented.

\section{Additional rationale for placement of pulse oximetry}

The use of pulse oximetry in aiding diagnosis of acute compartment syndrome has been observed previously and recommended in the monitoring of lower limb perfusion for prolonged lithotomy position (Tuckey 1996). However, the presence of a seemingly normal oximetry signal and saturation does not necessarily imply adequate perfusion (Tuckey 1996). It may be the case that reduced perfusion of the limb is not significant enough to affect the pulse oximetry until significant damage has already occurred. Nevertheless, in cases where the cannula site is not visible or regularly accessible, then pulse oximetry of the limb may still function as an important early warning system and the first sign of vascular compromise.

For many anaesthetic assistants it is common practice to place the pulse oximeter on the opposite side to the blood pressure cuff so as not to interrupt a continuous pulse oximetry trace. However, this case provides an additional rationale for the placement of the pulse oximeter on the side of the PIV cannula unless not clinically indicated.

Any vascular compromise to the limb caused by extravasation will eventually lead to damping of the pulse oximetry trace precipitated by impaired venous return leading to a reduction in the detectable arterial saturation. It is commonly recommended that the site of the pulse oximeter should be checked at least every two hours to avoid damage to the skin. Providing this is done with some regularity as is common practice in paediatrics this still leaves sufficient time for a serious extravasation injury to occur. However, in this case, the extravasation and compartment syndrome occurred within the first 30 minutes in theatre and may not have been detected until much later by routine pulse oximeter observations.

Wrap-around pulse oximetry probes are designed for long-term use and commonly used in the Paediatric Intensive Care Unit (PICU) as they are less likely to cause pressure sores or burns. This makes them more appropriate for use when it may not be possible to check the application site at regular intervals. Special effort should be made to establish visualisation of the cannula site as well as checking the site at regular intervals. If this is not possible then a pulse oximeter should be placed on a digit on the same side as the cannula site and practitioners should be aware of this additional rationale for oximetry placement. 


\section{Conclusion}

In summary, extravasation leading to acute compartment syndrome is a rare but serious complication of cannulation. Anaesthetic staff should be aware of the additional rationale for the placement of a pulse oximeter on paediatric patients as this may reduce the severity of an extravasation injury. This incident demonstrates that serious complications can occur in a very short time period in cases where the cannulation site is not easily visible or accessible. This case also functions as another example where multiple incisions combined with the squeeze technique and irrigation have successfully resolved extravasation caused vascular compromise. Fortunately the child suffered from no long-term effects or complications and required no further intervention following recovery from surgery and treatment for his extravasation injury.

\section{Competing Interests}

The authors declare that they have no competing interests.

\section{About the authors}

Daniel Rodger

BSc (Hons), MA (Ethics), RODP

Lecturer, Perioperative Practice, London South Bank University

Jacinda Hammerschlag

BSc, DA(SA), FCA(SA)

Consultant, Paediatric Anaesthetist, Guy's and St Thomas' NHS Foundation Trust, London

\section{References}

Al-Benna S, O'Boyle C, Holley J 2013 Extravasation Injuries in Adults International Scholarly Research Network Dermatology doi:10.1155/2013/856541

Behr AU 2015 Perioperative Care in Paediatric Orthopaedic Surgery. In: Astuto M, Ingelmo PM (ed) Perioperative Medicine in Pediatric Anesthesia Cham, Springer

Doellman D, Hadaway L, Bowe-Geddes LA et al 2009 Infiltration and Extravasation: Update on Prevention and Management Journal of Infusion Nursing 32 (4) 203-211

Driscoll C, Langer M, Burke S et al 2015 Improving Detection of IV Infiltrates in Neonates British Medical Journal Quality Improvement Reports 4 doi:

10.1136/bmjquality.u204253.w3874 
Duckworth AD, Porter DE, Ralston SH 2017 Orthopaedics, Trauma and Rheumatology, $2^{\text {nd }}$ edn, Edinburgh, Elsevier

Garg LR, Chhabra S, Singla GK, Lakhwani S 2015 Acute Compartment Syndrome of the Forearm in a Patient Undergoing Coronary Artery Bypass Surgery Indian Journal of Anaesthesia 59 (6) 387-388

Goutos I, Cogswell LK, Giele H 2014 Extravasation Injuries: A Review The Journal of Hand Surgery 39 (8) 808-818

Hakan S, Özer H 2007 Compartment Syndrome in the Hand Due to Extravasation of Contrast Material Archives of Orthopaedic and Trauma Surgery 127 (6) 425-427

Major TW, Huey TK 2016 Decreasing IV Infiltrates in the Pediatric Patient - System-Based Improvement Project Pediatric Nursing 42 (1) 14-20

Paquette V, McGloin R, Northway T et al 2011 Describing Intravenous Extravasation in Children The Canadian Journal of Hospital Pharmacy 64(5) 340-345

Philips LD, Gorski LA 2014 Manual of I.V. Therapeutics: Evidence-Based Practice for Infusion Therapy, $6^{\text {th }}$ edn, Philadelphia F.A. Davis Company

Reynolds PM, MacLaren R, Mueller SW et al 2015 Management of Extravasation Injuries: A Focused Evaluation of Noncytotoxic Medications Pharmacotherapy: The Journal of Human Pharmacology and Drug Therapy 34(6) 617-632

Treadwell T 2012 The Management of Intravenous Infiltration Injuries in Infants and Children Ostomy Wound Management 58 (7) 40-44

Tripi PA, Thomas S, Clebone A et al 2016 Peripheral Intravenous Catheter Problems in Infants and Children Presenting for Anesthesia and Surgery Middle East Journal of Anesthesiology 23 (4) 411-414

Tuckey J 1996 Bilateral Compartment Syndrome Complicating Prolonged Lithotomy Position British Journal of Anaesthesia 77 (4) 546-549 